\title{
EXPANSION PROPERTIES FOR FINITE SUBDIVISION RULES
}

II

\author{
WILLIAM FLOYD, WALTER PARRY, AND KEVIN M. PILGRIM
}

\begin{abstract}
We prove that every sufficiently large iterate of a Thurston map which is not doubly covered by a torus endomorphism and which does not have a Levy cycle is isotopic to the subdivision map of a finite subdivision rule. We determine which Thurston maps doubly covered by a torus endomorphism have iterates that are isotopic to subdivision maps of finite subdivision rules. We give conditions under which no iterate of a given Thurston map is isotopic to the subdivision map of a finite subdivision rule.
\end{abstract}

\section{INTRODUCTION}

This paper continues our study of expansion properties for finite subdivision rules begun in [12]. In [12] we usually began with a Thurston map which is the subdivision map of a finite subdivision rule. Here we begin with a general Thurston map, and we ask whether it is isotopic to the subdivision map of a finite subdivision rule.

An answer to the question of which Thurston maps are isotopic to subdivision maps of finite subdivision rules remains out of reach. A much more tractable problem is to determine which Thurston maps have iterates which are isotopic to subdivision maps of finite subdivision rules. Passage to an iterate does not materially affect dynamics, so in terms of dynamics, passage to an iterate is not very restrictive.

Our main result, Theorem 3.1 is that every sufficiently large iterate of a Thurston map which is not doubly covered by a torus endomorphism and which does not have a Levy cycle is isotopic to the subdivision map of a finite subdivision rule. A key ingredient in our proof of this is a result [1, Theorem C] of Bartholdi and Dudko, which implies that every Thurston map which is not doubly covered by a torus endomorphism and which does not have a Levy cycle is Böttcher expanding. In our proof of Theorem 3.1] what we really use is the fact that the map is Böttcher expanding. In Theorem 4.1 we handle those Thurston maps which are doubly covered by torus endomorphisms. So the question for iterates is answered except for Thurston maps which are not covered by torus endomorphisms and which have Levy cycles. Of course, many of these are subdivision maps of finite subdivision rules. However, in Theorem 5.1 we present a condition on such a Thurston map $f$ which implies that no iterate of $f$ is isotopic to the subdivision map of a finite subdivision rule. Section [5] concludes with an example of such a Thurston map.

Received by the editors August 20, 2019, and, in revised form, November 19, 2019.

2010 Mathematics Subject Classification. Primary 37F10, 52C20; Secondary 57M12.

Key words and phrases. Finite subdivision rule, expanding map, postcritically finite, Thurston map.

The third author was supported by Simons grant \#245269. 
We conclude this introduction with a brief history of this problem. There are two sources of motivation: one from geometry, another from dynamics. On the geometric side, finite subdivision rules were introduced in [4] as simplifications of patterns (informally described as "local replacement rules" or "finite replacement rules") observed when studying the action of cocompact Kleinian groups on the Riemann sphere; see e.g. [5, Section 3]. On the dynamical side, given a topological dynamical system, it is natural to ask whether there are Markov partitions with good topological properties. Thus the problem naturally arises to determine which Thurston maps are subdivision maps of finite subdivision rules. Independent solutions of this problem for all sufficiently large iterates of postcritically finite rational maps with no periodic critical points (they all are) were obtained almost simultaneously by Bonk-Meyer and Cannon-Floyd-Parry. The latter solution appeared as Theorem 1 in [6]. The former solution appeared as Corollary 15.2 in [3], where the result was generalized to expanding Thurston maps. The main result of [7] is that almost every Lattès rational map is the subdivision map of a finite subdivision rule with one tile type of a very special form. In [13, Theorem 1.2] Gao, Haïssinsky, Meyer, and Zeng prove that every sufficiently large iterate of a postcritically finite rational map whose Julia set is a Sierpiński carpet is the subdivision map of a finite subdivision rule. More recently Cui, Gao, and Zeng [9] proved that any critically finite rational map has an iterate that preserves a finite connected graph containing the postcritical set and thus is the subdivision map of a finite subdivision rule. Thus although the present work applies to more Thurston maps, its conclusion is weaker than those above because its conclusion only gives that the iterate is isotopic to a map which is a subdivision map. Thus it would be interesting to know if the techniques of [9] can be adapted to Böttcher expanding maps.

\section{Definitions AND PRELIMINARY LEMmas}

We fix some notation, make some definitions, and prove four lemmas. The notation and definitions which we introduce now will hold throughout this section. Let $f: S^{2} \rightarrow S^{2}$ be a Thurston map which is not doubly covered by a torus endomorphism and which does not have a Levy cycle.

Böttcher expanding maps. Let $P_{f}$ be the postcritical set of $f$, and let $P_{f}^{\infty}$ be the set of periodic points in $P_{f}$ whose cycles contain a critical point. Following [2. Definition 4.1] we say that $f$ is Böttcher expanding if (i) there exists a complete length metric on $S^{2}-P_{f}^{\infty}$ which is expanded by $f$, in the sense that for every nonconstant rectifiable compact curve $\gamma:[0,1] \rightarrow S^{2}-P_{f}^{\infty}$, the length of any lift of $\gamma$ under $f$ is strictly less than the length of $\gamma$, and (ii) for each $p \in P_{f}^{\infty}$, if $n$ is the least period of $p$, then the first-return map $f^{n}$, near $p$, is locally holomorphically conjugate to $z \mapsto z^{ \pm k}$ near the origin, where $k=\operatorname{deg}\left(f^{n}, p\right)$. Any two different choices of such local holomorphic coordinates differ by a multiplicative constant which is a $(k-1)$ st root of unity.

For example, if $f$ is rational, then according to [10 there is a canonically associated orbifold structure on $S^{2}$ whose singular points are precisely the elements of $P_{f}$, with elements of $P_{f}^{\infty}$ having weight infinity, so that these points correspond to punctures. The constant curvature metric on the orbifold universal cover descends to a metric which is then expanded by $f$; see [15, Appendix A]. 
Our point of departure for our subsequent analysis is the following result. As usual, the case of maps $f$ whose orbifold $\mathcal{O}_{f}$ is Euclidean (parabolic) requires special treatment. Among these, those with three or fewer postcritical points are isotopic to rational maps, by Thurston's characterization [10, and these are Böttcher expanding. The remaining types - those whose orbifolds have signature $(2,2,2,2)$ - are the exceptions in our setting. Any such map $f$ is isotopic through maps agreeing on $P_{f}$ to a map $g$ which is affine in the natural Euclidean orbifold structure and which lifts under the natural orbifold double-cover to an affine map of a torus induced by an affine map of the plane of the form $x \mapsto A_{f} x+b$.

The characterization in case (1) below is a deep result of Bartholdi and Dudko, announced in [1, Theorem C] and proved in [2, Theorem $\mathrm{A}=$ Theorem 4.4].

Theorem 2.1 (Expanding conditions). Suppose $f$ is a Thurston map.

(1) If $\mathcal{O}_{f}$ does not have signature $(2,2,2,2)$, then $f$ is isotopic to a smooth Böttcher expanding map $g$ if and only if it has no Levy cycles.

(2) If $\mathcal{O}_{f}$ has signature $(2,2,2,2)$, then $f$ is isotopic to a smooth Böttcher expanding map $g$ if and only if the eigenvalues of $A_{f}$ lie outside the closed unit disk.

The characterization in case (2) is elementary. (Sufficiency is clear. To prove necessity, note that an eigenvalue inside the closed unit disk must be real; a curve given by a segment in the corresponding eigenspace and containing a fixed point will have inverse images which do not get shorter under backward iteration, and this is an obstruction to the existence of some length metric which is contracted under taking preimages.)

So in the remainder of this section we may, and do, assume that $f$ itself is smooth and Böttcher expanding.

We will need uniform expansion. To accomplish this, we will excise open forwardinvariant neighborhoods, round-shaped in the natural conjugating coordinates, of points in $P_{f}^{\infty}$ to obtain a compact planar subset $\mathcal{M}$ with the property that its preimage $f^{-1}(\mathcal{M})$ is contained in the interior of $\mathcal{M}$. From compactness and expansion it then follows that $f$ is uniformly expanding on $\mathcal{M}$ in the sense that for some $0<\rho<1$ and for every nonconstant rectifiable compact curve $\gamma:[0,1] \rightarrow \mathcal{M}$, the length of any lift of $\gamma$ under $f$ is at most $\rho$ times the length of $\gamma$.

Fatou and Julia sets. Suppose $f$ is a Böttcher expanding smooth Thurston map and $\mathcal{M}$ is the complement of neighborhoods of the attractors as in the previous subsection. We recall here some facts from [2, Section 4.2].

The Julia set $\mathcal{J}_{f}$ is the closure of the set of repelling periodic points; a periodic point is repelling if there is no neighborhood $U \neq S^{2}$ with $f^{k}(U)$ compactly contained in $U$ for some $k>0$. The Fatou set $\mathcal{F}_{f}$ is the set of points at which the map $S^{2} \ni z \mapsto\left(z, f(z), f^{2}(z), \ldots\right) \in\left(S^{2}\right)^{\infty}$ is continuous. By [2, Lemma 4.6], $S^{2}=\mathcal{J}_{f} \sqcup \mathcal{F}_{f}$, and an equivalent formulation of $\mathcal{J}_{f}$ is that it coincides with the set of points which do not converge under iteration to the attracting cycles in $P_{f}^{\infty}$, i.e., with the set of points which never escape $\mathcal{M}$.

Notation. Let $\mathbb{D}$ be the open unit disk in $\mathbb{C}$. We use an overline to denote the topological closure of a set. So $\overline{\mathbb{D}}$ is the closed unit disk. An arc is the image of a closed unit interval under a homeomorphism; arcs are always closed. Let 
$d: S^{2} \times S^{2} \rightarrow \mathbb{R}$ be a spherical metric. All distances considered between points in $S^{2}$ are relative to $d$.

For each $p \in P_{f}^{\infty}$, the immediate basin of attraction $F_{p}$ of $p$ is the component of the Fatou set that contains $p$. Then for each $p \in P_{f}^{\infty}$, there is a homeomorphism $\psi_{p}: \mathbb{D} \rightarrow F_{p}$ with the following property. If $p \in P_{f}^{\infty}, q=f(p)$ and $k$ is the local degree of $f$ at $p$, then $\psi_{q}^{-1}\left(f\left(\psi_{p}(z)\right)\right)=z^{ \pm k}$ for every $z \in \mathbb{D}$. Moreover, the DouadyHubbard proof [11, Section 3.5] of the local connectivity of the filled Julia set of a subhyperbolic polynomial carries over in this setting, and so each $\psi_{p}$ extends to a continuous map $\psi_{p}$ : $\overline{\mathbb{D}} \rightarrow \bar{F}_{p}$. For more information, see the discussion before Lemma 4.7 in [2] or Theorem 4.14 of Milnor's paper [17].

The images in $\bar{F}_{p}$ of radii in $\overline{\mathbb{D}}$ are called rays, and the images of circles in $\mathbb{D}$ concentric about 0 are called equipotentials; these are independent of the choice of local holomorphic coordinates. The ray $\left\{\psi_{p}\left(r e^{i \theta}\right): 0 \leq r \leq 1\right\}$ with angle $\theta$ is said to land at $\psi_{p}\left(e^{i \theta}\right)$. For every nonnegative integer $n$, the connected components of $f^{-n}\left(F_{p}\right)$ are called Fatou components. Just as for rational Thurston maps which are Böttcher expanding, the Julia set of $f$ is connected, and each Fatou component is homeomorphic to a disk and is eventually periodic. Because $f$ is Böttcher expanding, there exists a finite cover of $S^{2} \backslash \bigcup_{p \in P_{f}^{\infty}} F_{p}$ by open sets $U_{i}$ such that the diameters of the connected components of the sets $f^{-n}\left(U_{i}\right)$ tend to 0 as $n \rightarrow \infty$.

Preliminary lemmas. We continue with four lemmas. The first lemma is a result in combinatorial topology about connecting vertices in a tiling of the disk. The next three lemmas are finiteness results about points in the boundary of an immediate basin of attraction. We emphasize that the above notation and definitions remain in force throughout this section.

The first lemma is essentially Lemma 4 in [6], though in [6] we assume that the $\mathrm{CW}$ complex gives a regular tiling. In the setting of a CW complex with underlying space a surface, the closed 2-cells are called tiles. The assumption in the second sentence is nontrivial, since open 2-cells need not be Jordan domains, and so a tile might contain a vertex in its interior.

Lemma 2.2. Let $X$ be a closed topological disk with the structure of a $C W$ complex such that every tile of $X$ is a closed topological disk. Let $u_{1}, u_{2}$, and $v$ be any triple of distinct vertices of $X$ such that each is in the boundary of a tile of $X$. Then there is an arc in the 1-skeleton of $X$ which has initial point $u_{1}$, terminal point $u_{2}$, and contains $v$.

Proof. We prove Lemma 2.2 by induction on the number of tiles in $X$. If $X$ has only one tile, then $u_{1}, u_{2}$, and $v$ are on the boundary of a polygon, and the theorem is clear. Suppose that $n$ is a positive integer and that the result holds if $X$ has $n$ tiles. Let $Y$ be a closed topological disk which has the structure of a $\mathrm{CW}$ complex with $n+1$ tiles such that every tile of $Y$ is a closed topological disk. Let $u_{1}, u_{2}, v$ be distinct vertices of $Y$ such that each is in the boundary of a tile of $Y$. Because every tile of $Y$ is a closed topological disk, $Y$ has distinct tiles $t_{1}$ and $t_{2}$ such that the closures of $Y \backslash t_{1}$ and $Y \backslash t_{2}$ are topological disks. Let $t$ be one of these tiles such that $v$ is in the closure $X$ of $Y \backslash t$. If $u_{1}, u_{2} \in X$, then we are done by induction. Now suppose that $u_{1} \in X$ but $u_{2} \notin X$. Let $w \neq u_{1}$ be one of the two points of $t \cap X \cap \partial Y$. By induction there is an arc in $X$ with endpoints $u_{1}$ and $w$ which contains $v$. This arc can easily be extended to $u_{2}$. The case in which $u_{1} \notin X$ and $u_{2} \in X$ is symmetric, and the case in which $u_{1}, u_{2} \notin X$ is similar. 
The second lemma shows that there cannot be infinitely many rays landing at a preperiodic point in the boundary of an immediate basin of attraction.

Lemma 2.3. Let $p \in P_{f}^{\infty}$, and let $q$ be a preperiodic point in $\partial F_{p}$. Then only finitely many rays in $\bar{F}_{p}$ land at $q$.

Proof. By replacing $f$ by an iterate of $f$, we may assume that $f(p)=p$ and $f(q)=q$. Let $\mathcal{R}$ be the set of rays in $\bar{F}_{p}$ which land at $q$.

We now define an equivalence relation $\sim$ on $\mathcal{R}$. Let $R_{1}, R_{2} \in \mathcal{R}$. If $R_{1}=R_{2}$, then $R_{1} \sim R_{2}$. Suppose that $R_{1} \neq R_{2}$. Then $R_{1} \cup R_{2}$ is a simple closed curve. We set $R_{1} \sim R_{2}$ if one of the connected components of $S^{2} \backslash\left(R_{1} \cup R_{2}\right)$ contains no element of $P_{f}$. This defines an equivalence relation on $\mathcal{R}$. Because $P_{f}$ is finite, this equivalence relation has only finitely many equivalence classes $\mathcal{R}_{1}, \ldots, \mathcal{R}_{j}$.

If $P_{f}$ contains just two points, then $f$ is Thurston equivalent to $z \mapsto z^{ \pm \operatorname{deg}(f)}$, and the lemma is true in this case. So we assume that $P_{f}$ contains a point other than $p$ and $q$. Hence if $R, S \in \mathcal{R}$ with $R \neq S$ and $R \sim S$, then exactly one connected component of $S^{2} \backslash(R \cup S)$ contains no element of $P_{f}$.

Let $R \in \mathcal{R}$. Then $f^{n}(R) \in \mathcal{R}$ for every nonnegative integer $n$. If the $j+1$ rays $R, f(R), f^{2}(R), \ldots, f^{j}(R)$ are not distinct for every $R \in \mathcal{R}$, then $\mathcal{R}$ is finite. So we may assume that $R$ is chosen so that these $j+1$ rays are distinct.

One of the equivalence classes $\mathcal{R}_{1}, \ldots, \mathcal{R}_{j}$ must contain at least two of these $j+1$ rays. We may assume that this equivalence class is $\mathcal{R}_{1}$. So there exist $R_{1}, R_{2} \in \mathcal{R}_{1}$ with $R_{1} \neq R_{2}$ such that some iterate of $f$ takes $R_{2}$ to $R_{1}$. We replace $f$ by this iterate and assume that $f\left(R_{2}\right)=R_{1}$.

Now let $D$ be the closed topological disk bounded by $R_{1} \cup R_{2}$ whose interior contains no element of $P_{f}$. The interior of $D$ is evenly covered by $f$. Since $f\left(R_{2}\right)=$ $R_{1}$, the disk $D$ therefore has a lift to a disk $\widetilde{D}$ whose boundary is the union of $R_{2}$ and a ray $R_{3}$ in $\bar{F}_{p}$ which lands at $q$. Then $f\left(R_{3}\right)=R_{2}$ and $R_{3} \in \mathcal{R}_{1}$ because the interior of $\widetilde{D}$ contains no element of $P_{f}$. Furthermore, the closure of the connected component of $S^{2} \backslash\left(R_{1} \cup R_{3}\right)$ which contains no element of $P_{f}$ contains $R_{2}$.

This construction can be repeated. We obtain an infinite sequence $R_{1}, R_{2}, R_{3}, \ldots$ of rays in $\mathcal{R}_{1}$ such that $f\left(R_{n+1}\right)=R_{n}$ for every positive integer $n$. Furthermore, the closure of the connected component of $S^{2} \backslash\left(R_{1} \cup R_{n}\right)$ which contains no element of $P_{f}$ contains $R_{2}, \ldots, R_{n-1}$ for every integer $n \geq 3$. It follows that the rays $R_{1}, R_{2}, R_{3}, \ldots$ converge to a ray in $\mathcal{R}$ fixed by $f$. The proof of Lemma 18.12 in Milnor's book [16] finally implies that if $\mathcal{R}$ contains a ray fixed by $f$, then only finitely many rays in $\bar{F}_{p}$ land at $q$.

This completes the proof of Lemma 2.3

The third lemma is about how many points in the boundaries of two immediate basins of attraction are accessible to a point that is in neither of their closures.

Lemma 2.4. Let $p$ and $q$ be distinct elements of $P_{f}^{\infty}$, and let $x \in S^{2} \backslash\left(\bar{F}_{p} \cup \bar{F}_{q}\right)$. Then there exist at most two points $y \in \partial F_{p} \cap \partial F_{q}$ for which there exists an arc $\gamma$ in $S^{2}$ with endpoints $x$ and $y$ such that $\gamma \cap\left(\bar{F}_{p} \cup \bar{F}_{q}\right)=\{y\}$.

Proof. Suppose that there are three distinct such points, $y_{1}, y_{2}, y_{3}$. For $i \in\{1,2,3\}$, let $\rho_{i}$ be a ray in $\bar{F}_{p}$ which joins $p$ and $y_{i}$, and let $\sigma_{i}$ be a ray in $\bar{F}_{q}$ which joins $q$ and $y_{i}$. Then $S^{2} \backslash\left(\bigcup_{j=1}^{3}\left(\rho_{j} \cup \sigma_{j}\right)\right)$ has three connected components. So the set $\bigcup_{j=1}^{3}\left(\rho_{j} \cup \sigma_{j}\right)$ separates $x$ from one of the points $y_{1}, y_{2}, y_{3}$.

This contradiction proves Lemma 2.4. 


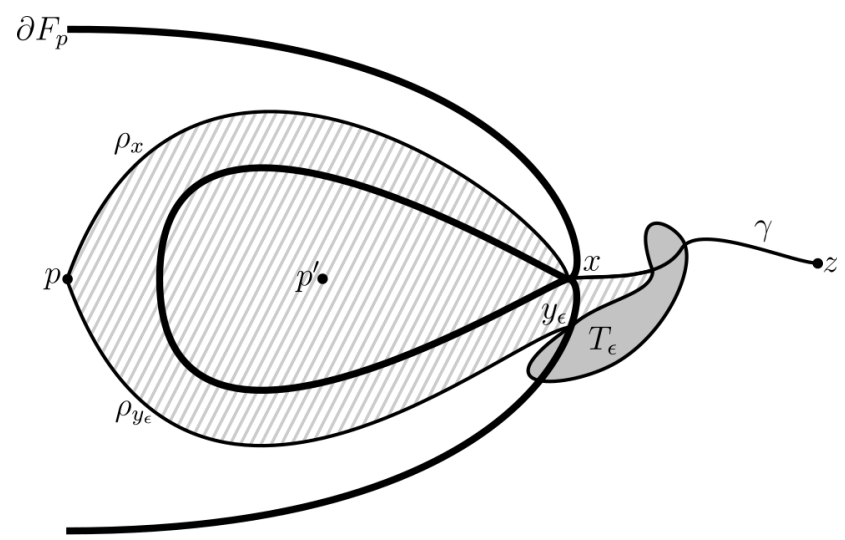

Figure 1. The situation in Lemma 2.5. The point $p^{\prime}$ is the solid black dot in the middle.

Here is the fourth lemma. Figure 1illustrates one of the finitely many situations in question. The region shaded by line segments is a connected component of the complement of the union of $\rho_{x}, \rho_{y_{\epsilon}}, T_{\epsilon}$, and $\gamma \backslash\{z\}$. Its interior contains a point $p^{\prime} \in P_{f}$.

Lemma 2.5. Let $p \in P_{f}^{\infty}$, and let $C$ be a connected component of $S^{2} \backslash \bar{F}_{p}$. Then there exist only finitely many points $x \in \partial F_{p}$ for which the following conditions are satisfied.

(1) There is a nontrivial arc $\gamma$ in $S^{2}$ with one endpoint $x$ whose other points are in $C$. Let $z$ be the endpoint of $\gamma$ other than $x$.

(2) There exist real numbers $\epsilon>0$ accumulating at 0 such that for each of them there exists a path connected set $T_{\epsilon}$ in $S^{2} \backslash\{z\}$ whose diameter is less than $\epsilon$ containing a point $y_{\epsilon} \in \partial F_{p}$ and a point of $\gamma$.

(3) There exist rays $\rho_{x}$ and $\rho_{y_{\epsilon}}$ in $\bar{F}_{p}$ which land at $x$ and $y_{\epsilon}$ such that the union of $\rho_{x}, \rho_{y_{\epsilon}}, T_{\epsilon}$, and $\gamma \backslash\{z\}$ separates $z$ from an element of $P_{f}$.

Proof. Let $x$ be a point in $\partial F_{p}$ which satisfies the conditions of the lemma. The compactness of $S^{2}, \gamma$, and $\bar{F}_{p}$ and condition (2) imply that a sequence of the sets $\overline{T_{\epsilon}}$ converges in the Hausdorff topology to a point in $\gamma \cap \partial F_{p}$. This point must be $x$. Using condition (3), we see that by passing to a subsequence we may assume that the corresponding sequence of rays $\rho_{y_{\epsilon}}$ converges to a ray $\rho_{x}^{\prime}$ which lands at $x$ such that $\rho_{x} \cup \rho_{x}^{\prime}$ is a simple closed curve which separates $C$ from an element of $P_{f}$. These simple closed curves separate $C$ from different elements of $P_{f}$ for different values of $x$. Since $P_{f}$ is finite, there are only finitely many such points $x$.

This proves Lemma 2.5.

\section{Realizability of Böttcher EXPANDing MAPS}

Theorem 3.1. Let $f$ be a Thurston map such that (i) if it does not lift to a torus endomorphism, it has no Levy cycles and (ii) if it lifts to a torus endomorphism, the associated affine map has both eigenvalues outside the unit circle. Then every sufficiently large iterate of $f$ is isotopic to the subdivision map of a finite subdivision rule. That is, if $f$ satisfies (i) and (ii), then every sufficiently large iterate 
of $f$ is isotopic to a Thurston map which admits an invariant connected graph whose vertex set contains the postcritical points of $f$.

Before giving the proof, we discuss the strategy of the proof and the dependence of the constants that arise in the proof. By Theorem 2.1 we may assume that $f$ is Böttcher expanding. The first thing we do is identify three subsets $P_{f}^{\infty}, P_{f}^{1}$, and $P_{f}^{0}$ that form a partition of the postcritical set $P_{f}$. The idea is to construct a graph, $G \subseteq S^{2}$, invariant up to isotopy and with vertex set containing $P_{f}$. For this we construct three finite sets $\mathcal{A}, \mathcal{B}, \mathcal{C}$ of curves in $S^{2}$. The sets $\mathcal{B}, \mathcal{C}$ consist of arcs, while $\mathcal{A}$ consists of closed curves which are either simple or trivial (a point); the latter are the elements of $P_{f}^{0}$. Our first approximation of $G$ as a set is the union of the curves in $\mathcal{A} \cup \mathcal{B} \cup \mathcal{C}$.

Our first constant $\rho \in(0,1)$ is chosen sufficiently close to 1 based on an open cover $\left\{U_{i}\right\}$ of $S^{2} \backslash \bigcup_{p \in P_{f}^{\infty}} F_{p}$ that exists because $f$ is Böttcher expanding. Once we have $\rho$, we construct the collection $\mathcal{A}$ of elements of $P_{f}^{0}$ and of equipotential curves corresponding to radius $\rho$, one for every $p \in P_{f}^{\infty}$. The set $\mathcal{B}$ consists of tails of rays. For every $p \in P_{f}^{\infty}$ the set $\mathcal{B}$ contains the tails from the equipotential of $F_{p}$ in $\mathcal{A}$ to an element $q \in P_{f}^{1} \cap \partial F_{p}$.

Our second constant $\delta$ is a sufficiently small positive real number such that $d(p, x)>4 \delta$ if $p \in P_{f}^{0}$ and $x$ is either in $P_{f}^{0}$ with $x \neq p$ or $x$ is in the closure of an immediate basin of attraction. The set $\mathcal{C}$ is a finite collection of arcs joining curves in $\mathcal{A}$ which are carefully chosen to satisfy ten conditions; the second and third conditions depend on $\delta$.

Having constructed the sets $\mathcal{A}, \mathcal{B}$, and $\mathcal{C}$, we give $S^{2}$ the structure of a cell complex $S$ whose 1 -skeleton is the union of the curves in $\mathcal{A}, \mathcal{B}$, and $\mathcal{C}$. We obtain a cell complex $f^{-n}(S)$ for every nonnegative integer $n$. Tiles in $f^{-n}(S)$ which are mapped by some iterate of $f$ into immediate basins of attraction are called Fatou tiles. The other tiles of $f^{-n}(S)$ are Julia tiles.

We next choose a sufficiently small real number $\epsilon \in(0, \delta)$. The number $\epsilon$ is chosen to give certain disjointness properties of $\epsilon$-neighborhoods of the $\operatorname{arcs}$ in $\mathcal{C}$ and to also satisfy one additional constraint coming from one of the ten conditions. Having chosen $\epsilon$, we use the Böttcher expanding property to choose a sufficiently large positive integer $N$ such that if $n \geq N$, then every Julia tile in $F^{-n}(S)$ has diameter less that $\epsilon$.

Now suppose that $n$ is a positive integer with $n \geq N$. We enlarge $\mathcal{A} \cup \mathcal{B} \cup \mathcal{C}$ to get the final form of $G$ by adding equipotential curves and rays in the immediate basins of attraction. We add enough of these so that every Fatou tile in $f^{-n}(S)$ has diameter less than $\epsilon$. Finally, we show that $f^{n}$ is isotopic rel $P_{f}$ to the subdivision map of a finite subdivision rule whose 1-skeleton is $G$.

Proof. By Theorem 2.1 we may assume that $f$ is Böttcher expanding. We partition the postcritical set $P_{f}$ of $f$ into three subsets. The subset $P_{f}^{\infty}$ consists of those periodic elements of $P_{f}$ whose cycles contain critical points. We let

$$
P_{f}^{1}=\left\{p \in P_{f}: p \in \partial F_{q}, q \in P_{f}^{\infty}\right\} \quad \text { and } \quad P_{f}^{0}=\left\{p \in P_{f}: p \notin \bigcup_{q \in P_{f}^{\infty}} \bar{F}_{q}\right\} .
$$

We intend to construct a finite subdivision rule $\mathcal{R}$. This involves equipping $S^{2}$ with the structure of a CW complex $S_{\mathcal{R}}$. The complex $S_{\mathcal{R}}$ will be defined as a subdivision of a CW complex $S$. We will define $S$ by means of its 1 -skeleton, which 
is a union of curves. These curves belong to three finite sets of curves $\mathcal{A}, \mathcal{B}$, and $\mathcal{C}$. The curves in $\mathcal{A}$ are either trivial, just points, or simple closed curves. The curves in $\mathcal{B}$ and $\mathcal{C}$ are nontrivial arcs.

We construct the set of curves $\mathcal{A}$ in this paragraph. It depends on a real parameter $\rho$ with $0<\rho<1$. Let $\mathbb{D}_{\rho}=\{z \in \mathbb{C}:|z|<\rho\}$. For every $p \in P_{f}^{\infty}$ let $D_{p}=\psi_{p}\left(\mathbb{D}_{\rho}\right) \subseteq F_{p}$. Recall that we have open sets $U_{i}$ whose existence is a consequence of the fact that $f$ is Böttcher expanding. We choose and fix $\rho$ so that the open disks $D_{p}$ together with the open sets $U_{i}$ cover $S^{2}$. The simple closed curves in $\mathcal{A}$ are those curves of the form $\partial D_{p}$ for $p \in P_{f}^{\infty}$. The trivial curves in $\mathcal{A}$ are the points in $P_{f}^{0}$. This completes the construction of $\mathcal{A}$.

Now we construct the set $\mathcal{B}$. If $P_{f}^{1}=\emptyset$, then $\mathcal{B}=\emptyset$. Suppose that $P_{f}^{1} \neq \emptyset$. Let $p \in P_{f}^{1}$. Then there exists $q \in P_{f}^{\infty}$ such that $p \in \partial F_{q}$. For every such point $q$ and for every ray $R$ in $\bar{F}_{q}$ which lands at $p$, we require that $\mathcal{B}$ contain the subarc of $R$ which has one endpoint $p$ and whose other endpoint is in $\partial D_{q}$. This completes the construction of $\mathcal{B}$. Lemma 2.3 implies that $\mathcal{B}$ is a finite set of arcs.

We next construct the set $\mathcal{C}$. For this we choose a real number $\delta>0$ such that $d(p, x)>4 \delta$ if $p \in P_{f}^{0}$ and $x \in P_{f}^{0} \cup\left(\bigcup_{q \in P_{f}^{\infty}} \bar{F}_{q}\right)$ with $x \neq p$. For every $x \in S^{2}$, let $B_{r}(x)$ be the open ball of radius $r>0$ about $x$. The set $\mathcal{C}$ will be constructed as a set of arcs $\gamma$ which satisfy the following conditions.

(1) The endpoints of every arc $\gamma$ are in distinct curves in $\mathcal{A}$, and one of these endpoints is in $\bigcup_{p \in P_{f}^{\infty}} \partial D_{p}$ if $P_{f}^{\infty} \neq \emptyset$.

(2) If $p \in P_{f}^{0}$ and $p \in \gamma$, then $\gamma \cap \partial B_{\delta}(p)$ and $\gamma \cap \partial B_{2 \delta}(p)$ each contain exactly one point.

(3) If $p \in P_{f}^{0}$ and $p \notin \gamma$, then $\gamma \cap \bar{B}_{2 \delta}(p)=\emptyset$.

(4) If $p \in P_{f}^{\infty}$ and $\gamma \cap \partial D_{p} \neq \emptyset$, then $\gamma \cap \bar{F}_{p}$ is a subarc of a ray.

(5) If $p \in P_{f}^{\infty}$ and $\gamma \cap \partial D_{p} \neq \emptyset$, then $\gamma$ avoids the finitely many points in $\partial F_{p}$ which satisfy the conditions of Lemma 2.5] relative to the connected component of $S^{2} \backslash \bar{F}_{p}$ which contains $\gamma \backslash \bar{F}_{p}$.

(6) If $p \in P_{f}^{\infty}$ and $\gamma \cap \partial D_{p}=\emptyset$, then $\gamma \cap \bar{F}_{p}=\emptyset$.

(7) If $\beta \in \mathcal{B}$, then $\gamma \cap \beta=\emptyset$.

(8) Two such arcs $\gamma$ may meet only at a point of $P_{f}^{0}$.

(9) Every element of $P_{f}^{0}$ is an endpoint of exactly two of the $\operatorname{arcs} \gamma$.

(10) The connected components of the complement in $S^{2}$ of the union of the curves in $\mathcal{A}, \mathcal{B}$, and $\mathcal{C}$ are open topological disks whose closures are closed topological disks.

Let $G$ be the union of the curves in $\mathcal{A}$ and $\mathcal{B}$. As we construct $\operatorname{arcs}$ in $\mathcal{C}$, we adjoin them to $G$. So $G$ will grow during this construction. It will eventually become a graph.

If $P_{f}^{\infty}=\emptyset$, then $G=P_{f}=P_{f}^{0}$. In this case we construct the $\operatorname{arcs}$ in $\mathcal{C}$ so that their union $G$ is a simple closed curve. It is easy to do this and satisfy conditions (1)-(10) .

So suppose that $P_{f}^{\infty} \neq \emptyset$. Figure 2 contains a schematic diagram of one possibility for $G$ after the construction of $\mathcal{C}$ is complete. The large dots are in $P_{f}^{0}$. The small dots are in $P_{f}^{1}$. The circles are the simple closed curves in $\mathcal{A}$. The line segments are the $\operatorname{arcs}$ in $\mathcal{B}$. The remaining arcs are the $\operatorname{arcs}$ in $\mathcal{C}$ which we are about to construct. 


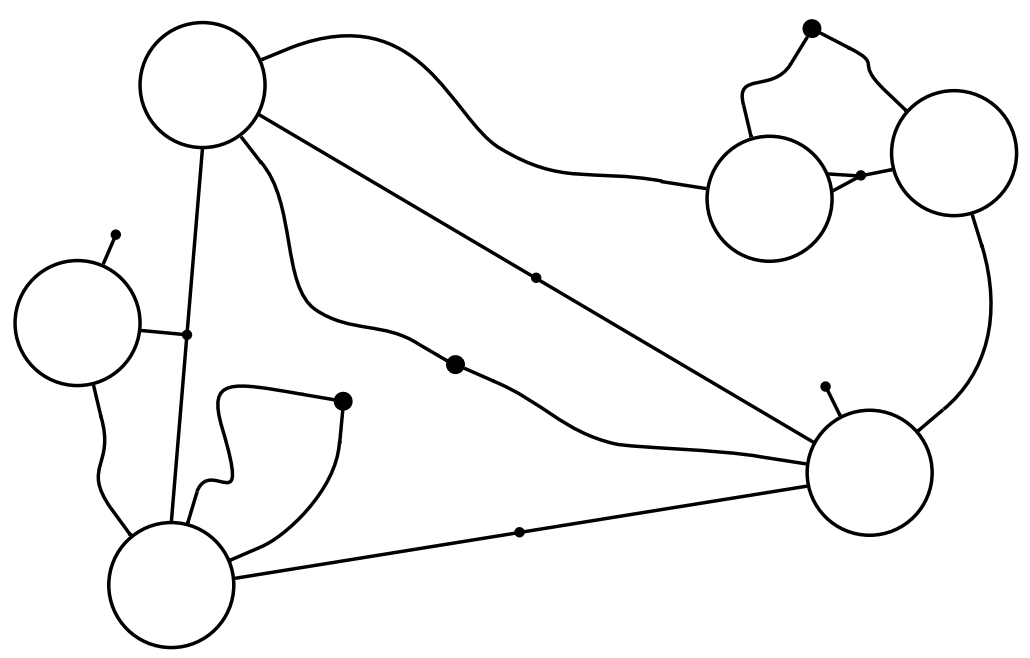

Figure 2. The union of the curves in $\mathcal{A}, \mathcal{B}$, and $\mathcal{C}$, a precursor of the final graph

Let $p \in P_{f}^{0}$. It is easy to construct an $\operatorname{arc} \alpha$ with one endpoint $p$, one endpoint in $\bigcup_{q \in P_{f}^{\infty}} \partial D_{q}$, disjoint from $P_{f} \backslash\{p\}$ and satisfying conditions (2), (3), (5), and (7). The arc $\alpha$ has a minimal subarc $\gamma$ which joins $p$ and $\bigcup_{q \in P_{f}^{\infty}} \bar{F}_{q}$. Lemma 2.4 implies that by choosing $\alpha$ to avoid finitely many points, we may assume that the endpoint of $\gamma$ other than $p$ lies in just one of the sets $\partial F_{q}$ with $q \in P_{f}^{\infty}$. We then extend $\gamma$ along a ray in $\bar{F}_{q}$ to $\partial D_{q}$. Now $\gamma$ satisfies conditions (1)-(7). We construct two such arcs $\gamma$ for every $p \in P_{f}^{0}$ so as to satisfy conditions (1)-(9). We put these arcs in $\mathcal{C}$ and adjoin them to $G$. After doing this for every element of $P_{f}^{0}$, every connected component of $G$ contains $\partial D_{p}$ for some $p \in P_{f}^{\infty}$.

Suppose that there exist $p, q \in P_{f}^{\infty}$ such that $\partial D_{p}$ and $\partial D_{q}$ lie in different connected components of $G$. We partition $P_{f}^{\infty}$ into two disjoint nonempty subsets $Q_{1}$ and $Q_{2}$ such that if $p \in Q_{1}$ and $q \in Q_{2}$, then $\partial D_{p}$ and $\partial D_{q}$ lie in different connected components of $G$. Let $H_{i}=\bigcup_{q \in Q_{i}} \bar{F}_{q}$ for $i \in\{1,2\}$.

Suppose that $H_{1} \cap H_{2}$ is an infinite set. Then there exist $p \in H_{1}$ and $q \in H_{2}$ such that $\bar{F}_{p} \cap \bar{F}_{q}$ is an infinite set. Now we repeatedly apply Lemma 2.4 to $p$, $q$, and $x \in P_{f}^{\infty} \backslash\{p, q\}$ to conclude that there exists $y \in \partial F_{p} \cap \partial F_{q}$ such that $y \notin \partial F_{p^{\prime}} \cup P_{f}^{1}$. We choose a ray in $\bar{F}_{p}$ with endpoint $y$ and a ray in $\bar{F}_{q}$ with endpoint $y$. The union of these rays contains a subarc $\gamma$ with endpoints in $\partial D_{p}$ and $\partial D_{q}$. It is possible to choose $y$ so that $\gamma$ satisfies condition (5). Therefore conditions (1)-(9) are maintained by putting $\gamma$ in $\mathcal{C}$. We put $\gamma$ in $\mathcal{C}$ and adjoin it to $G$. This reduces the number of connected components of $G$.

Suppose that $H_{1} \cap H_{2}$ is a finite set. Then we construct an arc $\alpha$ which joins $H_{1}$ and $H_{2}$ and which avoids $H_{1} \cap H_{2}$, all arcs in $\mathcal{B} \cup \mathcal{C}$ already constructed, and all closed balls $\bar{B}_{2 \delta}(p)$ with $p \in P_{f}^{0}$. The arc $\alpha$ has a minimal subarc $\gamma$ which joins $\bar{F}_{p}$ and $\bar{F}_{q}$ with $p \in Q_{1}$ and $q \in Q_{2}$. We may choose $\alpha$ so that $\gamma$ satisfies condition (5). Using Lemma 2.4 we find that $\alpha$ may also be chosen so that $p$ and $q$ are unique. We extend $\gamma$ along rays in $\bar{F}_{p}$ and $\bar{F}_{q}$ to an arc with endpoints in $\partial D_{p}$ and $\partial D_{q}$ 
while satisfying conditions (1)-(9). We put this $\operatorname{arc}$ in $\mathcal{C}$ and adjoin it to $G$. This reduces the number of connected components of $G$.

After constructing finitely many arcs as above, the set $G$ is connected. So the connected components of $S^{2} \backslash G$ are open topological disks. Let $D$ be one of these disks, and suppose that $\bar{D}$ is not a closed topological disk. Then there exists a simple closed curve $\alpha$ in $\bar{D}$ with exactly one point not in $D$ which satisfies the following property. There exist $p, q \in P_{f}^{\infty}$ such that $\partial D_{p} \cap \bar{D} \neq \emptyset, \partial D_{q} \cap \bar{D} \neq \emptyset$, and $\alpha$ separates $\partial D_{p}$ from $\partial D_{q}$. Working as in the previous paragraph, we construct an arc $\gamma$ with interior in $D$ satisfying conditions (1)-(9) which joins $\partial D_{p^{\prime}}$ and $\partial D_{q^{\prime}}$, where $p^{\prime}$ and $q^{\prime}$ are elements of $P_{f}^{\infty}$ such as $p$ and $q$.

In this way, we can eventually satisfy every condition (1)-(10). This completes the construction of $\mathcal{C}$.

We now have $\mathcal{A}, \mathcal{B}$, and $\mathcal{C}$. The union $G$ of their curves contains $P_{f}^{0}$ and $P_{f}^{1}$ but not $P_{f}^{\infty}$. We make $G$ into a 1-dimensional $\mathrm{CW}$ complex (graph) in the straightforward way: we declare that its vertices are the endpoints of the $\operatorname{arcs}$ in $\mathcal{B} \cup \mathcal{C}$. This in turn equips $S^{2}$ with the structure of a 2-dimensional CW complex $S$.

For every nonnegative integer $n$ we use $f^{n}$ to pull back $S$ to obtain a CW complex $f^{-n}(S)$. We call the closed 2-cells of such complexes tiles. We say that a tile is a Fatou tile if it is contained in some Fatou component, equivalently, if its image under some iterate of $f$ is contained in $F_{p}$ for some $p \in P_{f}^{\infty}$. Tiles which are not Fatou tiles are Julia tiles.

We next choose a real number $\epsilon$ with $0<\epsilon<\delta$. Conditions (2) and (3) imply that every arc $\gamma \in \mathcal{C}$ intersects $S^{2} \backslash \bigcup_{p \in P_{f}^{0}} B_{\delta}(p)$ in a subarc $\gamma^{*}$. We choose an open topological disk $D_{\gamma} \subseteq S^{2} \backslash P_{f}$ which contains $\gamma^{*}$ such that $D_{\gamma} \cap D_{\gamma^{\prime}}=\emptyset$ if $\gamma, \gamma^{\prime} \in \mathcal{C}$ with $\gamma \neq \gamma^{\prime}$. We also require that (i) $D_{\gamma} \cap \bar{F}_{p}=\emptyset$ if $p \in P_{f}^{\infty}$ such that $\gamma \cap \bar{F}_{p}=\emptyset$ and (ii) $D_{\gamma} \cap B_{2 \delta}(p)=\emptyset$ if $p \in P_{f}^{0}$ such that $p \notin \gamma$. We choose $\epsilon$ so that $D_{\gamma}$ contains the $\epsilon$-neighborhood of $\gamma^{*}$ for every $\gamma \in \mathcal{C}$.

We put one more restriction on $\epsilon$ in this paragraph. Condition (5) implies that $\epsilon$ may be chosen so that the following holds. Let $\gamma$ be any element of $\mathcal{C}$ which has an endpoint in $\partial D_{p}$ for some $p \in P_{f}^{\infty}$. Let $x$ be the point of $\gamma$ in $\partial F_{p}$, and let $z$ be the endpoint of $\gamma$ not in $\bar{F}_{p}$. Let $T$ be a path connected set in $S^{2} \backslash\{z\}$ with diameter less than $\epsilon$ which contains a point of $\gamma$ and a point $y \in \partial F_{p}$. Let $\rho_{x}$ and $\rho_{y}$ be rays in $\bar{F}_{p}$ which land at $x$ and $y$, respectively. Then the union of $\rho_{x}, \rho_{y}, T$, and $\gamma \backslash\{z\}$ does not separate $z$ from an element of $P_{f}$. This completes our choice of $\epsilon$.

We next choose a positive integer $N$. Recall that the assumptions and the choice of $\rho$ provide finitely many open sets $U_{i}$ which cover the Julia tiles of $S$ such that the diameters of the connected components of the sets $f^{-n}\left(U_{i}\right)$ tend to 0 as $n \rightarrow \infty$. We may refine this collection to a finite collection of open sets $V_{j}$ such that every $V_{j}$ is contained in some $U_{i}$, the $V_{j}$ 's cover the Julia tiles of $S$, and every $V_{j}$ intersects every Julia tile of $S$ in a connected set. It follows that there exists a positive integer $N$ such that the diameter of every Julia tile in $f^{-n}(S)$ is less than $\epsilon$ for every integer $n \geq N$.

We fix an integer $n \geq N$. We will prove that $f^{n}$ is isotopic to the subdivision map of a finite subdivision rule $\mathcal{R}$.

Now we define $S_{\mathcal{R}}$. Let $p \in P_{f}^{\infty}$. The disk $D_{p}$ is defined so that $D_{p}=\psi_{p}\left(\mathbb{D}_{\rho}\right)$. We subdivide $\mathbb{D}_{\rho}$ using radii and concentric circles as in Figure 3 in general with many radii and concentric circles, not necessarily uniformly spaced. We do this so 


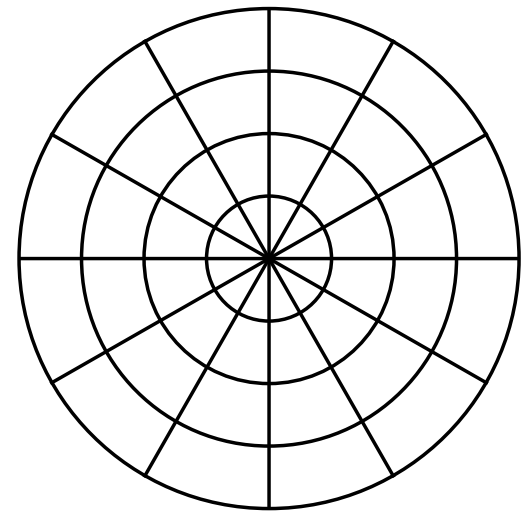

Figure 3. Subdividing $\mathbb{D}_{\rho}$

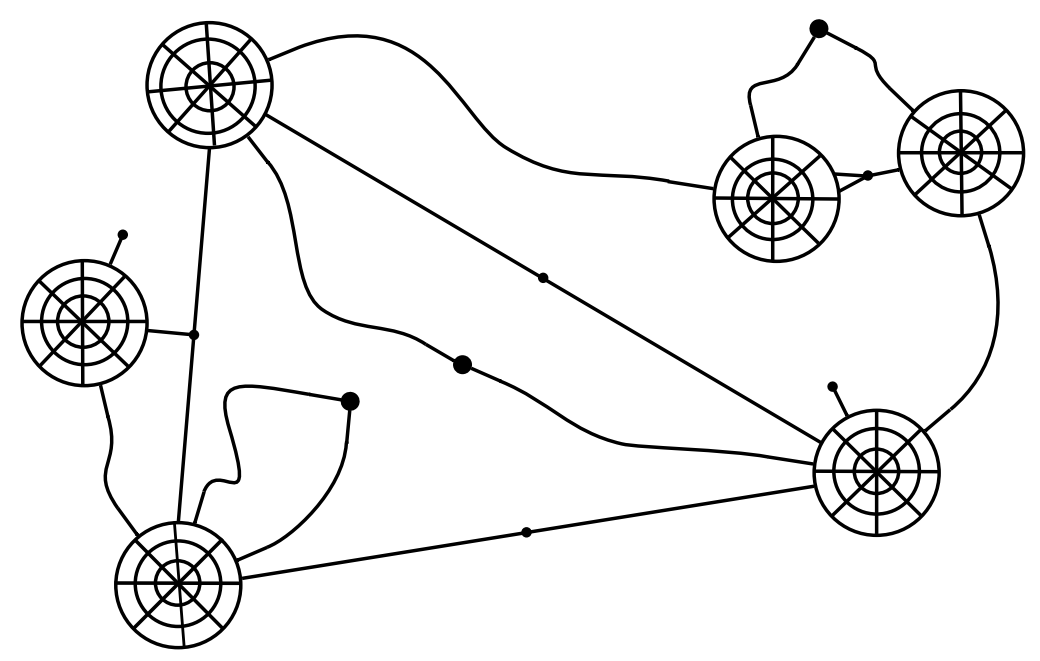

FiguRE 4. The 1-skeleton of the model subdivision complex $S_{\mathcal{R}}$

that the number of concentric circles is independent of $p$, and if an $\operatorname{arc}$ in $\mathcal{B} \cup \mathcal{C}$ has an endpoint $x \in \partial D_{p}$, then $\psi_{p}^{-1}(x)$ is not a vertex of this subdivision of $\mathbb{D}_{\rho}$. These subdivisions of $\mathbb{D}_{\rho}$, one for every $p \in P_{f}^{\infty}$, induce subdivisions of $S$ and $f^{-n}(S)$.

The Julia tiles in $f^{-n}(S)$ have diameters less than $\epsilon$. We choose these subdivisions of $\mathbb{D}_{\rho}$ so that every Fatou tile in the induced subdivision of $f^{-n}(S)$ also has diameter less than $\epsilon$. Thus every tile in the induced subdivision of $f^{-n}(S)$ has diameter less than $\epsilon$. We put one more restriction on these subdivisions. By possibly including more radii in these subdivisions, we may assume that there exists a positive integer $M$ such that the following condition holds. If $p \in P_{f}^{\infty}$, if $\gamma_{1}, \gamma_{2} \in \mathcal{B} \cup \mathcal{C}$ such that $\gamma_{i}$ has an endpoint $x_{i} \in \partial D_{p}$, and if one of the two arcs $\alpha \subseteq \partial D_{p}$ whose endpoints are $x_{1}$ and $x_{2}$ meets no other arcs in $\mathcal{B} \cup \mathcal{C}$, then $\alpha$ contains exactly $M$ vertices of the subdivision of $D_{p}$. The subdivision of $S$ obtained in this way is $S_{\mathcal{R}}$. See Figure 4 . 
It remains to prove that the 1-skeleton of $S_{\mathcal{R}}$ is isotopic rel $P_{f}$ to a subcomplex of the 1-skeleton of $f^{-n}\left(S_{\mathcal{R}}\right)$.

We begin the construction of an isotopy by moving only points near the arcs in $\mathcal{B}$. Let $p \in P_{f}^{1}$. Let $\beta$ be an $\operatorname{arc}$ in $\mathcal{B}$ which contains $p$. Then $\beta$ is a subarc of a ray in some $\bar{F}_{q}$ such that $p \in \partial F_{q}$. Moreover, $f^{n}(\beta)$ is a subarc of a ray in $\bar{F}_{f^{n}(p)}$, and $f^{n}(\beta)$ contains a unique arc $\beta^{\prime} \in \mathcal{B}$. So $\beta^{\prime}$ lifts via $f^{n}$ to a subarc $\gamma$ of $\beta$. So there exists a point pushing isotopy taking $\beta$ to $\gamma$ which fixes the complement of $F_{q}$ and even the complement of a small neighborhood of $\beta$ which avoids $q$. Because $p$ is the only element of $P_{f}$ in this neighborhood, this isotopy fixes $P_{f}$. Such isotopies, one for every arc in $\mathcal{B}$, can be combined into one isotopy which moves the arcs in $\mathcal{B}$ into the 1-skeleton of $f^{-n}\left(S_{\mathcal{R}}\right)$.

Now we prepare to move points near the $\operatorname{arcs}$ in $\mathcal{C}$. We define for every $p \in$ $P_{f}^{0} \cup P_{f}^{\infty}$ a subcomplex $W_{p}$ of $f^{-n}\left(S_{\mathcal{R}}\right)$ which is a closed topological disk with $p$ in its interior. If $p \in P_{f}^{\infty}$, then $W_{p}$ is the connected component of $f^{-n}\left(\bar{D}_{f^{n}(p)}\right)$ which contains $p$. Then $D_{p} \subseteq W_{p} \subseteq F_{p}$, and the boundary of $W_{p}$ is an equipotential in $F_{p}$. Now suppose that $p \in P_{f}^{0}$. Let $W_{p}$ be the smallest closed topological disk such that $W_{p}$ contains every tile of $f^{-n}\left(S_{\mathcal{R}}\right)$ which meets $\bar{B}_{\delta}(p)$. Since the diameters of the tiles of $f^{-n}\left(S_{\mathcal{R}}\right)$ are less than $\epsilon<\delta$, we have that $B_{\delta}(p) \subseteq W_{p} \subseteq B_{2 \delta}(p)$.

Let $\gamma \in \mathcal{C}$. We begin to define an arc $\widehat{\gamma}$ in the 1 -skeleton of $f^{-n}\left(S_{\mathcal{R}}\right)$ which is isotopic to $\gamma$. Let $x$ be an endpoint of $\gamma$. If $x \in P_{f}^{0}$, then set $p_{1}=x$. If $x \in \partial D_{p}$ for some $p \in P_{f}^{\infty}$, then set $p_{1}=p$. We define $p_{2}$ in the same way using the other endpoint of $\gamma$. Let $W$ be the subcomplex of $f^{-n}\left(S_{\mathcal{R}}\right)$ which as a set is the union of all tiles of $f^{-n}\left(S_{\mathcal{R}}\right)$ which meet $\gamma$ but are not contained in either $W_{p_{1}}$ or $W_{p_{2}}$. The choices of $\epsilon$ and $N$ imply that $W \subseteq D_{\gamma}$. The complexes $W_{p_{1}}$ and $W_{p_{2}}$ are constructed so that $\gamma \cap \partial W_{p_{1}} \neq \emptyset$ and $\gamma \cap \partial W_{p_{2}} \neq \emptyset$. So the 1 -skeleton of $W$ contains a minimal arc $\widehat{\gamma}$ which joins $\partial W_{p_{1}}$ and $\partial W_{p_{2}}$. If $i \in\{1,2\}$ and $p_{i} \in P_{f}^{\infty}$, then $\widehat{\gamma}$ meets $\partial F_{p_{i}}$ in exactly one point.

Suppose that $i \in\{1,2\}$ and that $p_{i} \in P_{f}^{0}$. We next use Lemma 2.2 to construct an extension of $\widehat{\gamma}$ to $p_{i}$. We take the complex $X$ in Lemma 2.2 to be $W_{p_{i}}$. Let $v=p_{i}$. Let $u_{1}$ be the unique vertex in $\partial W_{p_{i}} \cap \widehat{\gamma}$. The vertex $u_{2}$ is gotten in the same way using the other $\operatorname{arc}$ in $\mathcal{C}$ which contains $p_{i}$. We use the arc in Lemma 2.2 to extend $\widehat{\gamma}$ to an arc in the 1 -skeleton of $f^{-n}\left(S_{\mathcal{R}}\right)$ with endpoint $p_{i}$. This completes the definition of $\widehat{\gamma}$.

Now that we have $\widehat{\gamma}$, we wish to construct an isotopy rel $P_{f}$ which moves $\gamma$ to $\widehat{\gamma}$. Moreover, if $p_{i}=p \in P_{f}^{\infty}$ for either $i=1$ or $i=2$, then we wish to be able to extend this isotopy to the part of the 1-skeleton of $S_{\mathcal{R}}$ which is in $D_{p}$. To do this, we must deal with the difficulty that $\partial F_{p}$ might be complicated near $\gamma \cap \partial F_{p}$. In particular, the intersection of $F_{p}$ and the $\epsilon$-neighborhood of $\gamma$ might not be connected, as in Figure 5. Both $\gamma$ and $\widehat{\gamma}$ are drawn as thick arcs in Figure 5. The $\epsilon$-neighborhood of $\gamma$ is shaded dark gray. The rest of Figure 5 will be explained in the next paragraph.

With an eye toward Figure 5 we proceed as follows. We continue to assume that $p=p_{i} \in P_{f}^{\infty}$. Let $x$ and $y$ be the points at which $\gamma$ and $\widehat{\gamma}$ meet $\partial F_{p}$. Let $\rho_{x}$ and $\rho_{y}$ be the rays in $\bar{F}_{p}$ which land at $x$ and $y$ such that $\gamma$ contains the subarc of $\rho_{x}$ which joins $x$ and $\bar{D}_{p}$, while $\widehat{\gamma}$ contains the subarc of $\rho_{y}$ which joins $y$ and $W_{p}$. The latter subarc is in the boundary of a tile $t$ of $W$. We are in the situation of Lemma 2.5 with $T_{\epsilon}=t$ and $y_{\epsilon}=y$. Now we use the fact that $\gamma$ satisfies condition (5). It follows that there exists a closed topological disk $\Delta_{p} \subseteq S^{2}$ whose interior 


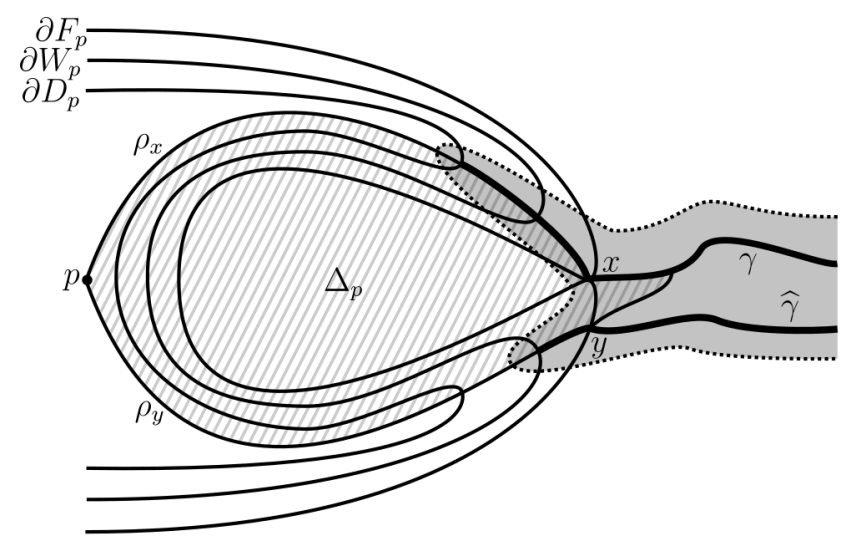

FIgURE 5. Constructing an isotopy which moves $\gamma$ to $\widehat{\gamma}$

contains no element of $P_{f}$ and whose boundary is contained in the union of $\rho_{x}, \rho_{y}$, $\gamma$, and $t$. Such a disk is shaded by line segments in Figure 5.

Now we choose an isotopy rel $P_{f}$ which moves $\gamma$ to $\widehat{\gamma}$. We choose it so that points in the middle section of $\gamma$ move within the smallest closed topological disk in $D_{\gamma}$ which contains $W$. At an end of $\gamma$ near $p=p_{i} \in P_{f}^{\infty}$, points of $\gamma$ move within $\Delta_{p}$. At an end of $\gamma$ near $p=p_{i} \in P_{f}^{0}$, points of $\gamma$ move within $B_{2 \delta}(p)$. We do this so that if $p=p_{i} \in P_{f}^{\infty}$, then $\rho_{x}$ moves to $\rho_{y}, D_{p}$ remains within $W_{p}$, and $W_{p}$ remains within $F_{p}$. These isotopies can be constructed compatibly with supports disjoint from the $\operatorname{arcs}$ in $\mathcal{B}$. We obtain an isotopy rel $P_{f}$ which moves the $\operatorname{arcs}$ in $\mathcal{B}$ and $\mathcal{C}$ into the 1-skeleton of $f^{-n}\left(S_{\mathcal{R}}\right)$.

We finally extend this isotopy to the entire 1-skeleton of $S_{\mathcal{R}}$. Let $p \in P_{f}^{\infty}$. Let $X$ be the intersection of $\bar{F}_{p}$ with the union of the $\operatorname{arcs}$ in $\mathcal{B} \cup \mathcal{C}$. We have an isotopy which moves the $\operatorname{arcs}$ in $\mathcal{B}$ and $\mathcal{C}$ to arcs comprising sets which we denote by $\mathcal{B}^{\prime}$ and $\mathcal{C}^{\prime}$. It also moves the cell complex $\bar{D}_{p}$ to a cell complex $\bar{D}_{p}^{\prime} \subseteq W_{p}$. Let $X^{\prime}$ be the intersection of $\bar{F}_{p}$ with the union of the arcs in $\mathcal{B}^{\prime} \cup \mathcal{C}^{\prime}$. We wish to construct an isotopy which fixes $p, S^{2} \backslash F_{p}$ and $X^{\prime}$ and which moves the 1-skeleton of $\bar{D}_{p}^{\prime}$ into the 1-skeleton of $W_{p}$. Figure 6 shows what we have from the point of view of $\overline{\mathbb{D}}$. It shows a portion of $\psi_{p}^{-1}\left(\partial W_{p}\right)$ and a portion of the cell structure of $\psi_{p}^{-1}\left(\bar{D}_{p}^{\prime}\right)$. The cell structure of $\psi_{p}^{-1}\left(W_{p}\right)$ is not shown. Suppose that $\psi_{p}^{-1}\left(\gamma_{1}\right)$ and $\psi_{p}^{-1}\left(\gamma_{2}\right)$ meet $\mathbb{D}_{\rho}$ in points $x_{1}$ and $x_{2}$. By construction, $x_{1}$ and $x_{2}$ are not vertices of $\mathbb{D}_{\rho}$. Let $x_{1}^{\prime}$ and $x_{2}^{\prime}$ be the points in $\psi_{p}^{-1}\left(\partial W_{p}\right)$ such that the isotopy which we have moves $\psi_{p}\left(x_{1}\right)$ and $\psi_{p}\left(x_{2}\right)$ to $\psi_{p}\left(x_{1}^{\prime}\right)$ and $\psi_{p}\left(x_{2}^{\prime}\right)$. So $\psi_{p}\left(x_{1}^{\prime}\right)$ and $\psi_{p}\left(x_{2}^{\prime}\right)$ are endpoints of two arcs in $X^{\prime}$. Suppose that one of the two $\operatorname{arcs} \alpha$ in $\psi_{p}^{-1}\left(\bar{D}_{p}^{\prime}\right)$ whose endpoints are $x_{1}^{\prime}$ and $x_{2}^{\prime}$ contains no other points of $\psi_{p}^{-1}\left(X^{\prime}\right)$. Let $\alpha^{\prime}$ be the corresponding arc in $\psi_{p}^{-1}\left(\partial W_{p}\right)$ with endpoints $x_{1}^{\prime}$ and $x_{2}^{\prime}$. We wish to construct an isotopy which moves $\alpha$ to $\alpha^{\prime}$, which fixes $x_{1}^{\prime}$ and $x_{2}^{\prime}$, and moves every vertex of $\psi_{p}^{-1}\left(\bar{D}_{p}^{\prime}\right)$ in $\alpha$ to a vertex of $\psi_{p}^{-1}\left(W_{p}\right)$. In order to do this, all that is needed is that the number of vertices of $\psi_{p}^{-1}\left(W_{p}\right)$ in $\alpha^{\prime}$ be at least as large as the number of vertices of $\psi_{p}^{-1}\left(\bar{D}_{p}^{\prime}\right)$ in $\alpha$. This is guaranteed by the fact that the subdivisions of $\mathbb{D}_{\rho}$ were chosen so that $\alpha$ has $M$ vertices and the number of vertices in $\alpha^{\prime}$ is a positive multiple of $M$. 


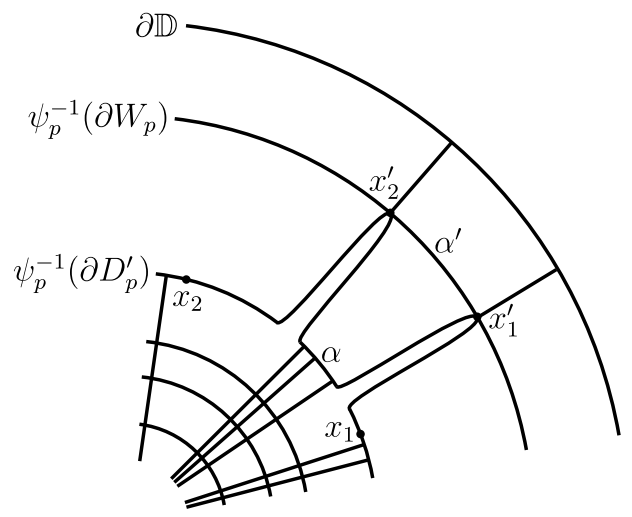

Figure 6 . The picture in $\overline{\mathbb{D}}$ after moving the $\operatorname{arcs}$ in $\mathcal{B}$ and $\mathcal{C}$

This allows us to isotop the radii of $\bar{D}_{p}^{\prime}$ into the radii of $W_{p}$ while fixing $X^{\prime}$ and $S^{2} \backslash F_{p}$. It is easy to isotop the equipotentials in $\bar{D}_{p}^{\prime}$ to the equipotentials in $W_{p}$ because they are equal in number.

Thus the isotopy rel $P_{f}$ which moves the arcs in $\mathcal{B}$ and $\mathcal{C}$ into the 1-skeleton of $f^{-n}\left(S_{\mathcal{R}}\right)$ can be extended to an isotopy rel $P_{f}$ which moves the 1-skeleton of $S_{\mathcal{R}}$ into the 1-skeleton of $f^{-n}\left(S_{\mathcal{R}}\right)$. This proves Theorem 3.1 .

\section{Realizability of $(2,2,2,2)$-Euclidean Thurston maps}

This section treats the case when the orbifold of $f$ has signature $(2,2,2,2)$, i.e., is isotopic to a map doubly covered by an affine torus map. Not every such map $f$ has an iterate isotopic to the subdivision map of a finite subdivision rule; Theorem 4.1 gives a characterization in terms of the eigenvalues of the associated linear map.

Our nonrealizability results in this section and the next are essentially based on the following statement. If a Thurston map $f$ is the subdivision map of a finite subdivision rule, then the lift of $f^{-1}$ to the universal covering orbifold of $f$ is combinatorially distance nonincreasing. This is formally stated in statement (1) of Lemma 6.1 of [12]. We use this result in this section, and in the next section we use its generalization to the universal covering space of the complement in $S^{2}$ of the postcritical set of $f$. We discuss this result in the next paragraph.

Let $f: S^{2} \rightarrow S^{2}$ be a Thurston map which is the subdivision map of a finite subdivision rule. Let $P_{f}$ be the postcritical set of $f$. Let $D$ be either the universal covering orbifold of $f$ or the universal covering space of $S^{2}-P_{f}$. Let $\pi: D \rightarrow S^{2}$ be the associated branched covering map. The multifunction $f^{-1}$ lifts to a genuine function $F: D \rightarrow D$, so that $f \circ \pi \circ F=\pi$. Because $f$ is the subdivision map of a finite subdivision rule, there exists a cell structure on $S^{2}$ and a refinement of it called its first subdivision such that $f$ maps interiors of cells of the first subdivision homeomorphically to interiors of cells of the initial cell structure. In general the original cell structure on $S^{2}$ does not lift to $D$, but only because of the need for some vertices "at infinity". So we enlarge $D$ to a space $D^{*}$ by adding appropriate vertices at infinity. We then construct a cell structure on $D^{*}$ by using $\pi$ to lift the initial cell structure on $S^{2}$. As a result, $\pi$ extends to a branched covering map $\pi: D^{*} \rightarrow S^{2}$. The first subdivision of this cell structure on $D^{*}$ is the lift of the 
first subdivision of the initial cell structure on $S^{2}$. The map $F$ also extends to $D^{*}$. It homeomorphically maps interiors of cells of the initial cell structure on $D^{*}$ to interiors of cells of its first subdivision. A fat path in $D^{*}$ is the union of all tiles which meet a given curve in $D$ (not $D^{*}$ ). The length of a fat path is 1 less than the number of these tiles. The fat path distance function on $D$ (not $D^{*}$ ) is defined so that the "distance" between points $x, y \in D$ is the minimum length of a fat path joining them. (The fat path function does not define a metric because points in the interior of a tile have zero distance from each other, and it doesn't define a pseudometric because a point in an edge or a vertex that is in more than one tile has positive distance from itself.) Because $F$ maps initial tiles of $D^{*}$ into initial tiles of $D^{*}$, it is distance nonincreasing with respect to this fat path distance function.

The main result of [7] is that almost every Lattès rational map is the subdivision map of a finite subdivision rule with one tile type of a very special form. The following theorem treats a larger class of Thurston maps which need not be rational. It almost completely determines which $(2,2,2,2)$-Euclidean Thurston maps are subdivision maps of finite subdivision rules. In this case, the orbifold universal cover is $\mathbb{R}^{2}$, and the map $F$ in the statement of the theorem is the inverse of the map $F$ of the previous paragraph.

Theorem 4.1. Suppose $f$ is a Thurston map whose orbifold $\mathcal{O}_{f}$ has signature $(2,2,2,2)$. Furthermore, suppose $f$ is normalized so that it has a lift to the plane of the form $F(x)=A x+b$, where $A$ is a $2 \times 2$ matrix of integers and $b$ is an integral linear combination of the columns of $A$.

(1) If both eigenvalues of $A$ have absolute value greater than 1, then every sufficiently large iterate of $f$ is the subdivision map of a finite subdivision rule.

(2) If \pm 1 is an eigenvalue of $A$, then $f$ is the subdivision map of a finite subdivision rule with one tile type.

(3) If $A$ has an eigenvalue with absolute value less than 1 , then no iterate of $f$ is Thurston equivalent to the subdivision map of a finite subdivision rule.

Remark. Theorem 3.1 of [7] implies that in the setting of Theorem 4.1] if in addition $f$ is rational, then some iterate of $f$ is the subdivision map of a subdivision rule with one tile type. The proof given there does not use the fact that $f$ is rational, only that it is expanding.

Proof. We begin by making the connection between $A$ and $f$ precise. Let $\Lambda$ be the sublattice of $\mathbb{Z}^{2}$ generated by the columns of $A$. Let $\Gamma$ be the group of all isometries of $\mathbb{R}^{2}$ generated by rotations of order 2 about the elements of $\Lambda$. The map $F$ respects the action of $\Gamma$ on $\mathbb{R}^{2}$ and induces a map on $\mathbb{R}^{2} / \Gamma$ in a canonical way. The latter space is homeomorphic to $S^{2}$. We conjugate this map on $\mathbb{R}^{2} / \Gamma$ to $S^{2}$ by this homeomorphism. The result is $f$. The image of $\Lambda$ in $S^{2}$ is the postcritical set $P_{f}$ of $f$, and the image of $\mathbb{Z}^{2} \backslash \Lambda$ in $S^{2}$ is the set of critical points of $f$. Since the postcritical set is invariant under $f, f\left(P_{f}\right)=P_{f}$, so $F(\Lambda) \subset \Lambda$. In particular $F(0) \in \Lambda$ and so $b \in \Lambda$. This verifies that the normalization condition can always be achieved.

Statement (1) follows from Theorem 3.1 


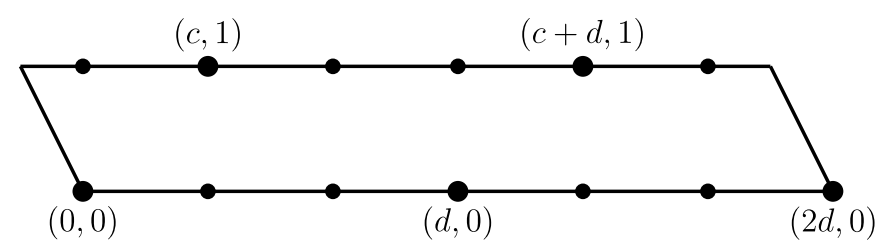

Figure 7. A fundamental domain $P$ for $\Gamma$ in the situation of statement $(2)$

To prove statement (2), suppose that \pm 1 is an eigenvalue of $A$. Replacing $A$ by $-A$ does not change $f$, so we assume that 1 is an eigenvalue of $A$. Let $d$ be the degree of $f$. Since the product of the eigenvalues of $A$ is $\operatorname{det}(A)=d$, the eigenvalues of $A$ are 1 and $d$.

We continue by finding a normal form for $A$. Since the eigenvalues of $A$ are rational numbers, $A$ has nonzero eigenvectors with rational entries, hence with integer entries. Let $(p, q)$ be an eigenvector of $A$ with eigenvalue $d$ such that $p$ and $q$ are relatively prime integers. Then there exist integers $r$ and $s$ such that $p s-q r=1$. So $(p, q)$ and $(r, s)$ form a basis of $\mathbb{Z}^{2}$. Since $(p, q)$ is an eigenvector with eigenvalue $d=\operatorname{det}(A)$, when we conjugate $A$ to this basis, we obtain a matrix of the form $\left[\begin{array}{ll}d & c \\ 0 & 1\end{array}\right]$. Since conjugation of $A$ by an element of $\mathrm{SL}(2, \mathbb{Z})$ corresponds to conjugation of $f$ by a homeomorphism, we may assume that $A=\left[\begin{array}{ll}d & c \\ 0 & 1\end{array}\right]$. (Conjugating this by a matrix of the form $\left[\begin{array}{ll}1 & a \\ 0 & 1\end{array}\right]$, we may even assume that $0 \leq c \leq d-2$.)

Let $P$ be the parallelogram in $\mathbb{R}^{2}$ bounded by the lines given by $y=0, y=1$, the 1-eigenspace of $A$, and the line parallel to this through $(2 d, 0)$. See Figure 7 where dots mark elements of $\mathbb{Z}^{2}$ and large dots mark elements of $\Lambda$. This parallelogram is a fundamental domain for $\Gamma$.

The image in $S^{2}$ of the bottom of $P$ is an arc $\alpha$ joining the postcritical points which are the images of $(0,0)$ and $(d, 0)$. Similarly, the image in $S^{2}$ of the top of $P$ is an arc $\beta$ joining the postcritical points which are the images of $(c, 1)$ and $(c+d, 1)$. The map $F$ maps horizontal lines through elements of $\mathbb{Z}^{2}$ to horizontal lines through elements of $\mathbb{Z}^{2}$. Hence $f$ stabilizes the union $\alpha \cup \beta$. The annulus in $S^{2}$ determined by $\alpha$ and $\beta$ is fibered by arcs which are the images of the line segments in $P$ parallel to the left and right sides of $P$. The map $f$ stabilizes the set of these arcs. The map $f$ also stabilizes the simple closed curve in $S^{2}$ which is the image of the line in $\mathbb{R}^{2}$ given by $y=1 / 2$. The degree of $f$ on this simple closed curve is $d>1$. Hence the Lefschetz number of $f$ restricted to this simple closed curve is not 0 , and so the Lefschetz fixed point theorem implies that $f$ fixes a point of this simple closed curve. Thus there exists an arc $\gamma$ joining $\alpha$ and $\beta$ which is stabilized by $f$. The union of the $\operatorname{arcs} \alpha, \beta$, and $\gamma$ is a tree containing the postcritical points of $f$ which is stabilized by $f$. This tree provides $S^{2}$ with the structure of a CW complex making $f$ the subdivision map of a finite subdivision rule with one tile type. This proves statement (2).

To prove statement (3), we argue by contradiction. Since the hypotheses are preserved under passing to an iterate, we may replace $f$ by an iterate and suppose that $A$ has an eigenvalue $\lambda$ less than 1 in absolute value and that $f$ is Thurston equivalent to the subdivision map $g: S^{2} \rightarrow S^{2}$ of a finite subdivision rule. Since the product of the eigenvalues of $A$ is the degree of $f$, the eigenvalues of $A$ are real, and 
the eigenvalue other than $\lambda$ has absolute value greater than 1 . Since multiplying $A$ by -1 does not affect $f$, we may furthermore assume that $\lambda>0$.

Because $f$ and $g$ are Thurston equivalent, there exist two homeomorphisms $h, h^{\prime}:\left(S^{2}, P_{f}\right) \rightarrow\left(S^{2}, P_{g}\right)$ such that $f h=h^{\prime} g$ with $h$ and $h^{\prime}$ isotopic rel $P_{f}$. By conjugating $g$, we may assume that $h^{\prime}=1$, so that $f$ and $g$ have the same postcritical set: $P_{f}=P_{g}$. Then $h\left(P_{f}\right)=P_{f}$, and $h$ is isotopic to the identity map rel $P_{f}$. Just as $f$ lifts to $F: \mathbb{R}^{2} \rightarrow \mathbb{R}^{2}$ via the branched covering map from $\mathbb{R}^{2}$ to $S^{2}$, the map $h$ has a lift $H: \mathbb{R}^{2} \rightarrow \mathbb{R}^{2}$. Because $h$ is isotopic to the identity map rel $P_{f}$, we may choose $H$ so that its restriction to $\Lambda$ is the identity map. Furthermore, because $S^{2}$ is compact, there exists a bound on the distances which $H$ moves the points of $\mathbb{R}^{2}$.

The results of the previous paragraph imply that the map $G=F \circ H$ lifts $g$. So $G^{-1}=H^{-1} \circ F^{-1}$ lifts the multifunction $g^{-1}$. Furthermore, there exists a positive real number $J$ such that $\left\|G^{-1}(x)-F^{-1}(x)\right\| \leq J$ for every $x \in \mathbb{R}^{2}$. We choose $J$ so large that we even have that $\left\|G^{-1}(x)-A^{-1} x\right\| \leq J$ for every $x \in \mathbb{R}^{2}$.

In this paragraph we prove that there exists a positive real number $K$ such that $\left\|A^{-n} w\right\| \leq K \lambda^{-n}\|w\|$ for every positive integer $n$ and every $w \in \mathbb{R}^{2}$. If $A$ is diagonal, then this is clear. In general, $A$ can be conjugated by an element of $\mathrm{GL}(2, \mathbb{R})$ to a diagonal matrix. Since the conjugating matrix deforms the standard metric on $\mathbb{R}^{2}$ by a bounded amount, the desired result follows.

Now let $L$ be the $\lambda$-eigenspace of $A$. We will obtain a contradiction by considering the action of the iterates of $G^{-1}$ on $L$. Let $x \in L$. Then $G^{-1}(x)=A^{-1} x+y_{1}=$ $\lambda^{-1} x+y_{1}$ for some $y_{1} \in \mathbb{R}^{2}$ with $\left\|y_{1}\right\| \leq J$. Similarly, $G^{-2}(x)=\lambda^{-2} x+A^{-1} y_{1}+y_{2}$ for some $y_{2} \in \mathbb{R}^{2}$ with $\left\|y_{2}\right\| \leq J$. Using the result of the previous paragraph, we inductively see for every positive integer $n$ that

$$
\begin{aligned}
\left\|G^{-n}(x)-\lambda^{-n} x\right\| & \leq J K\left(\lambda^{-n+1}+\cdots+\lambda^{-2}+\lambda^{-1}+1\right) \\
& =J K\left(\lambda^{-n}-1\right)\left(\lambda^{-1}-1\right)^{-1} .
\end{aligned}
$$

Hence

$$
\begin{aligned}
\lambda^{-n}\|x\| & =\left\|\lambda^{-n} x-\lambda^{-n} 0\right\| \\
& =\left\|\left(\lambda^{-n} x-G^{-n}(x)\right)+\left(G^{-n}(x)-G^{-n}(0)\right)+\left(G^{-n}(0)-\lambda^{-n} 0\right)\right\| \\
& \leq\left\|\lambda^{-n} x-G^{-n}(x)\right\|+\left\|G^{-n}(x)-G^{-n}(0)\right\|+\left\|G^{-n}(0)-\lambda^{-n} 0\right\| \\
& \leq 2 J K\left(\lambda^{-n}-1\right)\left(\lambda^{-1}-1\right)^{-1}+\left\|G^{-n}(x)-G^{-n}(0)\right\|,
\end{aligned}
$$

and so

$$
\begin{aligned}
\left\|G^{-n}(x)-G^{-n}(0)\right\| & \geq \lambda^{-n}\|x\|-2 J K\left(\lambda^{-n}-1\right)\left(\lambda^{-1}-1\right)^{-1} \\
& \geq\left(\|x\|-2 J K\left(\lambda^{-1}-1\right)^{-1}\right) \lambda^{-n} .
\end{aligned}
$$

Thus if $\|x\|>2 J K\left(\lambda^{-1}-1\right)^{-1}$, then $\left\|G^{-n}(x)-G^{-n}(0)\right\|$ tends to $\infty$ as $n$ tends to $\infty$.

We finally use the fact that $g$ is the subdivision map of a finite subdivision rule. Note that $G^{-1}$ is a lift of $g^{-1}$ to the universal covering orbifold of $g$. So the discussion at the beginning of this section applies. The initial tiling of $S^{2}$ lifts to a tiling $T$ of $\mathbb{R}^{2}$. Because $S^{2}$ is compact and this branched covering map is regular, the tiles of $T$ decompose into finitely many orbits under the action of its group of deck transformations. Because the elements of this group are Euclidean isometries, there is thus a bound on the diameters of the tiles of $T$. So a bound on the fat path distance between two points of $\mathbb{R}^{2}$ provides a bound on the Euclidean 
distance between these points. Hence the lack of a bound on the Euclidean distances $\left\|G^{-n}(x)-G^{-n}(0)\right\|$ implies the lack of a bound on the fat path distances between $G^{-n}(x)$ and $G^{-n}(0)$. This contradicts the observation made at the beginning of this section that $G^{-1}$ is distance nonincreasing with respect to the fat path distance function.

This proves Theorem 4.1.

\section{NonREALIZABILITY CONDITIONS}

Suppose $f$ is a Thurston map with postcritical set $P_{f}$. We denote by $\mathcal{C}$ the set of simple, unoriented, essential, nonperipheral (not homotopic into arbitrarily small neighborhoods of elements of $P_{f}$ ) curves in $S^{2}-P_{f}$, up to homotopy in $S^{2}-P_{f}$. We denote by $\odot$ the union of homotopy classes of inessential and peripheral curves in $S^{2}-P_{f}$. Via pullback, we obtain a pullback relation $\stackrel{f}{\leftarrow}$ on $\mathcal{C} \cup\{\odot\}$, where $\gamma \stackrel{f}{\leftarrow} \widetilde{\gamma}$ if and only if some component $\delta \subset f^{-1}(\gamma)$ is in $\widetilde{\gamma}$. It is natural to view this as a multivalued map of $\mathcal{C} \cup\{\odot\}$ to itself and to consider its orbits; a point has finite image under this map. Note that the image of $\odot$ under this multivalued map consists of only $\odot$. An orbit $\gamma_{0} \stackrel{f}{\leftarrow} \gamma_{1} \stackrel{f}{\leftarrow} \gamma_{2} \ldots$ is univalent if the unsigned degrees $\operatorname{deg}\left(f: \gamma_{i} \rightarrow \gamma_{i-1}\right), i=1,2, \ldots$, are all equal to 1 ; it is wandering if the $\gamma_{i}$ 's are all distinct and not equal to $\odot$.

Similarly, we denote by $\mathcal{A}$ the set of homotopy classes of arcs $\alpha:[0,1] \rightarrow S^{2}$ with $\alpha(\{0,1\}) \subset P_{f}, \alpha(t) \in S^{2}-P_{f}$ for $t \notin\{0,1\}$, up to homotopy through arcs with the same properties and reparameterizations which may reverse orientation, and subject to the condition that $\alpha$ is not peripheral in a similar sense (that is, $\alpha$ is not homotopic rel $P_{f}$ to a constant arc). Note that an element of $\mathcal{A}$ might join a point of $P_{f}$ to itself. We denote by $a$ the union of those homotopy classes of arcs $\alpha:[0,1] \rightarrow S^{2}$ defined similarly as for $\mathcal{A}$, but with $P_{f}$ replaced by $f^{-1}\left(P_{f}\right)$, and subject to the condition that they do not define elements of $\mathcal{A}$. Again by pullback we obtain a relation $\mathcal{A} \cup\left\{0_{-}\right\} \stackrel{f}{\leftarrow} \mathcal{A} \cup\{\circ-\}$ such that the corresponding multivalued map has finite images of points and $\circ$ maps to itself. The corresponding degrees by which arcs map are always equal to 1 . Wandering is defined similarly.

Theorem 5.1provides conditions under which no iterate of a given Thurston map is Thurston equivalent to the subdivision map of a finite subdivision rule.

Theorem 5.1. Let $f: S^{2} \rightarrow S^{2}$ be a Thurston map with postcritical set $P_{f}$. If the pullback relation on arcs has wandering orbits or if the pullback relation on curves has wandering univalent orbits, then no iterate of $f$ is Thurston equivalent to the subdivision map of a finite subdivision rule.

Remark. If $f$ is homotopic to a Böttcher expanding map, then (i) its pullback relation on arcs has no wandering orbits, and (ii) its pullback relation on curves has no univalent wandering orbits. Compare [19]. For maps without periodic branch points, similar and stronger results are shown in [14, Proposition 5, Theorem 8]. Fix some length metric as in the definition of Böttcher expanding.

To prove (ii), note that the length of any essential, simple, nonperipheral curve is bounded from below away from zero; that there are only finitely many such curves whose length is bounded above by some positive constant; and that if $f$ maps such a curve $\widetilde{\gamma}$ by degree 1 to another such curve $\gamma$ by degree 1 , then the definition of Böttcher expanding implies $\ell(\tilde{\gamma})<c \ell(\gamma)$ where $c<1$. 
To prove (i), we argue as follows. For $p \in P_{f}^{\infty}$ and $0<\rho<1$ denote by $D_{p, \rho}$ the disk of radius $\rho$ about $p$ in the local Böttcher coordinates. Define the complexity $C[\alpha]$ of a homotopy class of $\operatorname{arcs} \alpha$ to be the infimum, among homotopic representatives, of the length of the portion of the subarc lying outside the disks $D_{p, 1 / 2}$. There are only finitely many arc classes whose complexity is bounded above by some positive constant. Böttcher expansion implies that if $f$ maps such an arc $\widetilde{\alpha}$ to such an arc $\alpha$, then $C[\widetilde{\alpha}])<c C[\alpha]+\delta$ where $c<1$ is as in the previous paragraph and $\delta>0$ is the maximum, over such $p$, of the distance between $\partial D_{p, 1 / 2}$

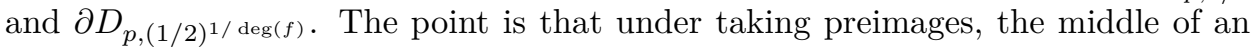
arc shortens by a definite multiplicative amount, while the end segments lengthen by a bounded additive amount. Combined, these two facts mean that there are no wandering arcs under iterated pullback.

Proof. Suppose $\alpha_{0} \stackrel{f}{\leftarrow} \alpha_{1} \stackrel{f}{\leftarrow} \cdots$ is such an orbit.

Every iterate of $f$ satisfies the assumptions of the theorem with the sequence $\alpha_{0}, \alpha_{1}, \alpha_{2}, \ldots$ replaced by a subsequence. Every Thurston map homotopic to $f$ satisfies the assumptions of the theorem with $\alpha_{0}, \alpha_{1}, \alpha_{2}, \ldots$ modified by homotopies rel $P_{f}$. Thus to prove the theorem, it suffices to prove that $f$ is not the subdivision map of a finite subdivision rule.

We prove this by contradiction. Suppose that $f$ is the subdivision map of a finite subdivision rule $\mathcal{R}$. We modify $\alpha_{0}$ by a homotopy rel $P_{f}$ so that, except for its endpoints in the case of arcs, for some nonnegative integer $n$ it meets the 1-skeleton of $\mathcal{R}^{0}\left(S^{2}\right)$ transversely in $n$ points which are not vertices. We then modify $\alpha_{1}, \alpha_{2}, \ldots$ so as to preserve the assumptions of the theorem. Because $f$ is the subdivision map of $\mathcal{R}$ and $f$ maps $\alpha_{i}$ bijectively to $\alpha_{i-1}$, it follows that, except for endpoints in the case of arcs, for every nonnegative integer $i, \alpha_{i}$ meets the 1-skeleton of $\mathcal{R}^{0}\left(S^{2}\right)$ transversely in at most $n$ points which are not vertices.

Because $\alpha_{0}, \alpha_{1}, \alpha_{2}, \ldots$ are mutually not homotopic rel $P_{f}$, the order of $P_{f}$ must be at least 4 . So the universal covering space of $S^{2} \backslash P_{f}$ is the open disk $D$. Let $\pi: D \rightarrow S^{2} \backslash P_{f}$ be the universal covering map. As at the beginning of Section 4 , we enlarge $D$ to a space $D^{*}$ by adding vertices at infinity and extend $\pi$ to a branched covering map $\pi: D^{*} \rightarrow S^{2}$. We use $\pi$ to lift the cell structure of $S^{2}$ to $D^{*}$.

Let $F$ be a fundamental domain in $D^{*}$ for $\pi$ which is a union of tiles of $D^{*}$. For every nonnegative integer $i$, let $\widetilde{\alpha}_{i}$ be a lift of $\alpha_{i}$ to $D^{*}$ which contains a point in the interior of $F$. Then each $\widetilde{\alpha}_{i}$ meets the interior of $F$ and crosses at most $n$ edges of $D^{*}$. Since there is a positive integer $N$ such that every tile of $D^{*}$ has at most $N$ vertices and edges, these lifts are contained in the union of finitely many translates of $F$ under the group of deck transformations of $\pi$. In the case of arcs, it follows that $\widetilde{\alpha}_{i}$ and $\widetilde{\alpha}_{j}$ have the same initial and terminal endpoints for some indices $i \neq j$. It follows that $\widetilde{\alpha}_{i}$ and $\widetilde{\alpha}_{j}$ are homotopic rel $\partial D^{*}$. Hence $\alpha_{i}$ and $\alpha_{j}$ are homotopic rel $P_{f}$, a contradiction. The case of simple closed curves is only slightly different.

This proves Theorem 5.1 .

Theorem 4.1 shows that some Thurston maps with Euclidean orbifolds are not subdivision maps of finite subdivision rules. We illustrate Theorem 5.1 with the following example of a Thurston map with hyperbolic orbifold such that no iterate is Thurston equivalent to the subdivision map of a finite subdivision rule.

Example 5.2. We begin by describing the subdivision map of a finite subdivision rule on $S^{2}$. The 1-skeleton of the initial cell structure on $S^{2}$ is a square with four 

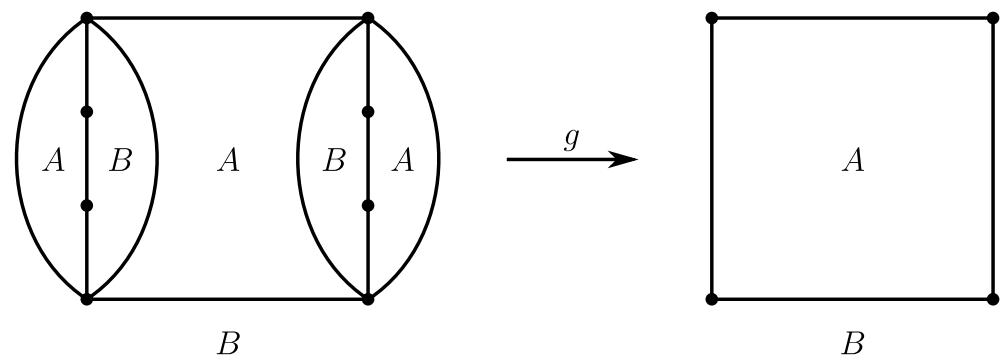

Figure 8. Defining the Thurston map $g$ for Example 5.2

vertices and four edges. There are two tiles, each a quadrilateral. The subdivision map $g$, a Thurston map, acts as in Figure 8, Figure 8 shows two copies of $S^{2}$, each with one point at $\infty$. The initial cell structure is shown in the right part of Figure 8 with the two tiles labeled $A$ and $B$. The map $g$ fixes all four initial vertices and the two horizontal edges pointwise. The tile labels in the left part indicate the initial tiles which are the images of these tiles. So $g$ has degree 3. Its local degree at every critical point is 2 . These four critical points are all fixed by $g$, and so they form the postcritical set of $g$.

Let $\tau$ be a Dehn twist, or even a half Dehn twist, about a simple closed curve $\gamma$ which is vertical relative to the orientation of Figure 8, Let $f=\tau^{n} \circ g$ for some nonzero integer $n$. Let $\alpha_{0}$ be the top edge of tile $A$. We lift $\alpha_{0}$ via the iterates of $f$ to obtain $\operatorname{arcs} \alpha_{1}, \alpha_{2}, \alpha_{3}, \ldots$ The arc condition of Theorem 5.1 is satisfied. So no iterate of $f$ is Thurston equivalent to the subdivision map of a finite subdivision rule. Note that $\{\gamma\}$ is a Levy cycle for $g$ and hence also for $f$.

The canonical decomposition of a Thurston map [19, Theorem 10.2] provides another source of examples.

Example 5.3. Suppose $f$ is an obstructed Thurston map with an elliptic piece in its decomposition that possesses at least four marked points. Then there exists a homeomorphism $h:\left(S^{2}, P_{f}\right) \rightarrow\left(S^{2}, P_{f}\right)$ such that the twist $h \circ f$ is not homotopic to the subdivision map of a finite subdivision rule.

One may arrange it so that $h$ is supported on the given elliptic piece $U$ and the first-return map of $h \circ f$ is pseudo-Anosov on $U$, so that every curve contained in this elliptic piece wanders under pullback of the restriction of $f$ to this piece.

A bit more concretely: the mating of the 1/4-rabbit quadratic polynomial with its complex conjugate provides an obstructed Thurston map $f_{0}$. Its fourth iterate $f_{1}=f_{0}^{4}$ has the property that it is homotopic to a map which is the identity on a subsurface $U \subset S^{2}-P_{f_{1}}$ such that $U$ is a sphere with four holes and the boundary of each hole is essential and nonperipheral in $S^{2}-P_{f_{1}}$. Setting $f=h \circ f_{1}$ where $h: U \rightarrow U$ is pseudo-Anosov and $\left.h\right|_{\partial U}=$ id will realize this construction.

\section{ACKNOWLEDGMENT}

We thank the referee for insightful comments and for suggestions on improving the exposition. 


\section{REFERENCES}

[1] Laurent Bartholdi and Dzmitry Dudko, Algorithmic aspects of branched coverings (English, with English and French summaries), Ann. Fac. Sci. Toulouse Math. (6) 26 (2017), no. 5, 1219-1296, DOI 10.5802/afst.1566. MR3746628

[2] Laurent Bartholdi and Dzmitry Dudko, Algorithmic aspects of branched coverings $I V / V$. Expanding maps, Trans. Amer. Math. Soc. 370 (2018), no. 11, 7679-7714, DOI 10.1090/tran/7199. MR3852445

[3] Mario Bonk and Daniel Meyer, Expanding Thurston maps, Mathematical Surveys and Monographs, vol. 225, American Mathematical Society, Providence, RI, 2017. MR3727134

[4] J. W. Cannon, W. J. Floyd, and W. R. Parry, Finite subdivision rules, Conform. Geom. Dyn. 5 (2001), 153-196, DOI 10.1090/S1088-4173-01-00055-8. MR1875951

[5] J. W. Cannon, W. J. Floyd, and W. R. Parry, Conformal modulus: the graph paper invariant or the conformal shape of an algorithm, Geometric group theory down under (Canberra, 1996), de Gruyter, Berlin, 1999, pp. 71-102. MR.1714840

[6] J. W. Cannon, W. J. Floyd, and W. R. Parry, Constructing subdivision rules from rational maps, Conform. Geom. Dyn. 11 (2007), 128-136, DOI 10.1090/S1088-4173-07-00167-1. MR.2329140

[7] J. W. Cannon, W. J. Floyd, and W. R. Parry, Lattès maps and finite subdivisison rules, Conform. Geom. Dyn. 14 (2010), 113-140, DOI 10.1090/S1088-4173-10-00203-1. MR2629972

[8] J. W. Cannon, W. J. Floyd, W. R. Parry, and K. M. Pilgrim, Nearly Euclidean Thurston maps, Conform. Geom. Dyn. 16 (2012), 209-255, DOI 10.1090/S1088-4173-2012-00248-2. MR2958932

[9] Guizen Cui, Yan Gao, and Jinsong Zeng, Invariant graphs of rational maps, https://arxiv . org/abs/1907.02870, 2019.

[10] Adrien Douady and John H. Hubbard, A proof of Thurston's topological characterization of rational functions, Acta Math. 171 (1993), no. 2, 263-297, DOI 10.1007/BF02392534. MR. 1251582

[11] A. Douady and J. H. Hubbard, Étude dynamique des polynômes complexes. Partie I (French), Publications Mathématiques d'Orsay [Mathematical Publications of Orsay], vol. 84, Université de Paris-Sud, Département de Mathématiques, Orsay, 1984. MR762431

[12] William J. Floyd, Walter R. Parry, and Kevin M. Pilgrim, Expansion properties for finite subdivision rules I, Sci. China Math. 61 (2018), no. 12, 2237-2266, DOI 10.1007/s11425-0169265-y. MR.3881956

[13] Yan Gao, Peter Haïssinsky, Daniel Meyer, and Jinsong Zeng, Invariant Jordan curves of Sierpiński carpet rational maps, Ergodic Theory Dynam. Systems 38 (2018), no. 2, 583-600, DOI 10.1017/etds.2016.47. MR3774834

[14] Peter Haïssinsky and Kevin M. Pilgrim, An algebraic characterization of expanding Thurston maps, J. Mod. Dyn. 6 (2012), no. 4, 451-476, DOI 10.3934/jmd.2012.6.451. MR.3008406

[15] Curtis T. McMullen, Complex dynamics and renormalization, Annals of Mathematics Studies, vol. 135, Princeton University Press, Princeton, NJ, 1994. MR.1312365

[16] John Milnor, Dynamics in one complex variable, Introductory lectures, Friedr. Vieweg \& Sohn, Braunschweig, 1999. MR1721240

[17] John Milnor, Hyperbolic components, with an appendix by A. Poirier, Conformal dynamics and hyperbolic geometry, Contemp. Math., vol. 573, Amer. Math. Soc., Providence, RI, 2012, pp. 183-232, DOI 10.1090/conm/573/11428. MR2964079

[18] Kevin Michael Pilgrim, Cylinders for iterated rational maps, ProQuest LLC, Ann Arbor, MI, Thesis (Ph.D.)-University of California, Berkeley, 1994. MR2691488

[19] Kevin M. Pilgrim, Combinations of complex dynamical systems, Lecture Notes in Mathematics, vol. 1827, Springer-Verlag, Berlin, 2003. MR2020454

[20] Nikita Selinger and Michael Yampolsky, Constructive geometrization of Thurston maps and decidability of Thurston equivalence, Arnold Math. J. 1 (2015), no. 4, 361-402, DOI 10.1007/s40598-015-0024-4. MR 3434502 
Department of Mathematics, Virginia Tech, Blacksburg, Virginia 24061

Email address: floyd@math.vt.edu

URL: http://www.math.vt.edu/people/floyd

Department of Mathematics and Statistics, Eastern Michigan University, Ypsilanti, MiCHIGAN 48197

Email address: walter.parry@emich.edu

Department of Mathematics, Indiana University, Bloomington, Indiana 47405

Email address: pilgrim@indiana.edu 